\section{AB0365 CLINICAL, LABORATORY AND IMAGING OUTCOMES IN TOCILIZUMAB-TREATED PATIENTS WITH LARGE VESSEL-GIANT CELL ARTERITIS ACCORDING TO EARLY ONSET THERAPY}

D. Prieto-Peña ${ }^{1}$, I. Martínez-Rodríguez ${ }^{2}$, B. Atienza-Mateo ${ }^{1}$, O. CuencaVera' $^{2}$, F. J. Gomez de la Fuente ${ }^{2}$, A. Sanchez-Salmón², M. A. GonzálezGay ${ }^{1}$, R. Blanco ${ }^{1}{ }^{1}$ Hospital Universitario Marques de Valdecilla, IDIVAL, Rheumatology, Santander, Spain; ${ }^{2}$ Hospital Universitario Marques de Valdecilla, IDIVAL, Nuclear Medicine, Santander, Spain

Background: Tocilizumab (TCZ) has shown efficacy in large vessel vasculitis (LVV)-Giant Cell Arteritis (LVV-GCA) (1-2). ${ }^{18} \mathrm{~F}$-fluodeoxyglucose positron emission tomography $\left({ }^{18} \mathrm{~F}\right.$-FDG PET/CT) is useful to assess LVV disease activity (35). It is unknown if early treatment with TCZ may have an influence on clinical, laboratory and imaging outcomes.

Objectives: To assess clinical, laboratory and PET/CT activity improvement in LVV-GCA patients treated with TCZ according to the time from disease diagnosis to TCZ onset.

Methods: Comparative single-center study of 30 LVV-GCA patients treated with TCZ who were divided into 2 groups depending on the time of onset of TCZ: a) early onset ( $\leq 6$ months; $n=15$ ) and $\mathbf{b}$ ) late onset ( $>6$ months; $n=15)$. All patients had a baseline and a follow-up PET/CT scan. Complete clinical improvement and normalization of laboratory markers (CRP $\leq 0.5 \mathrm{mg} / \mathrm{dL}$ and/or ESR $\leq 20 \mathrm{~mm} / 1^{\text {st }}$ hour) was assessed. For imaging evaluation, normalization of total visual score (TVS) was considered when TVS $=0$ and normalization of semiquantitative activity if the target to background ratio (TBR) at the thoracic aorta was $<1.34$.

Results: 30 patients were included (24 women/6 men); mean age $65.7 \pm 9.8$ years. Patients in the TCZ early-onset group were receiving higher doses of prednisone (10.0[5.9-15.0] vs 5.0 [5.0-7.5] mg/day; $p<0.01$ ) and had higher TVS scores (7.0 [4.0-9.0] vs 3.0 [2.0-5.0]; $p<0.01$ ) at baseline (Table 1). Following TCZ initiation, after a mean of $10.8 \pm 3.7$ months, most patients achieved complete clinical improvement and normalization of ESR and CRP in both groups. Uncoupling with imaging outcomes was observed in both groups. Although non-significant statistical differences were observed, complete TBR normalization $($ TBR $<1.34)$ and complete TVS normalization (TVS=0) tended to be more frequent in the group of patients who received early-onset TCZ therapy (Figure 1).

Table 1.

\begin{tabular}{|c|c|c|c|}
\hline & $\begin{array}{c}\text { Early-onset TCZ } \\
\text { therapy } \\
(n=15)\end{array}$ & $\begin{array}{c}\text { Late-onset TCZ } \\
\text { therapy } \\
(n=15)\end{array}$ & $\mathbf{p}$ \\
\hline \multicolumn{4}{|l|}{ General features } \\
\hline Age (years), mean $\pm S D$ & $65.8 \pm 9.9$ & $65.5 \pm 10.1$ & 0.94 \\
\hline Sex (female), n (\%) & $11(73.3)$ & $13(86.7)$ & 0.65 \\
\hline $\begin{array}{l}\text { GCA evolution before TCZ onset, median } \\
\text { [IQR] }\end{array}$ & $2.0[1.0-5.0]$ & $18.0[9.0-34.0]$ & $<0.01$ \\
\hline \multicolumn{4}{|l|}{ Laboratory } \\
\hline $\mathrm{ESR}$ (mm/1st hour), mean $\pm \mathrm{SD}$ & $34.7 \pm 26.3$ & $30.8 \pm 28.7$ & 0.70 \\
\hline CRP (mg/dL), median [IQR] & $1.1[0.6-2.3]$ & $0.8[1.8-2.5]$ & 0.28 \\
\hline $\begin{array}{l}\text { Prednisone dose (mg/day), mean } \pm \text { SD } \\
\text { TCZ therapy }\end{array}$ & $10.0[5.9-15.0]$ & $5.0[5.0-7.5]$ & 0.01 \\
\hline Intravenous, n (\%) & $10(66.7)$ & $11(73.3)$ & 0.99 \\
\hline Combined with MTX, $n(\%)$ & $6(40)$ & $8(53.3)$ & 0.46 \\
\hline \multicolumn{4}{|l|}{ PET /CT activity } \\
\hline TBR at thoracic aorta & $1.86 \pm 0.69$ & $1.54 \pm 0.18$ & 0.09 \\
\hline TVS & $7.0[4.0-9.0]$ & $3.0[2.0-5.0]$ & $<0.01$ \\
\hline Complete clinical improvement, $\mathrm{n}(\%)$ & $13(86.7)$ & $12(80)$ & 0.99 \\
\hline $\begin{array}{l}\text { Normalization of ESR and CRP, } n(\%) \\
\text { PET/CT improvement }\end{array}$ & $15(100)$ & $15(100)$ & 0.99 \\
\hline Complete TBR normalization & $6(40)$ & $3(20)$ & 0.23 \\
\hline Complete TVS normalization & $2(13.3)$ & $1(6.7)$ & 0.54 \\
\hline
\end{tabular}

CRP: C-reactive protein; ESR: erythrocyte sedimentation rate; TBR: target-to-background ratio * Normalization of TBR was considered when TBR $<1.34$. ${ }^{\star *}$ Normalization of TVS was considered when TVS $=0$.

Conclusion: TCZ was effective in patients with LVV-GCA regardless the time from disease diagnosis to TCZ onset. However, complete normalization of vascular activity in PET/CT scans tended to occur more likely in patients who receive early-onset TCZ therapy within the first 6 months of the disease.

REFERENCES:

[1] Calderón-Goercke M et al. Semin Arthritis Rheum. 2019; 49:126-135. PMID: 30655091

[2] Prieto Peña D et al. Clin Exp Rheumatol. 2020. PMID: 33253103

[3] González-Gay MA et al. Expert Rev Clin Immunol. 2018; 14:593-605. PMID: 29877748

[4] Martínez-Rodríguez et al. Semin Arthritis Rheum.2018; 47(4): 530-537. PMID: 28967430
[5] Prieto-Peña D et al. Semin Arthritis Rheum. 2019; 48:720-727. PMID 28967430

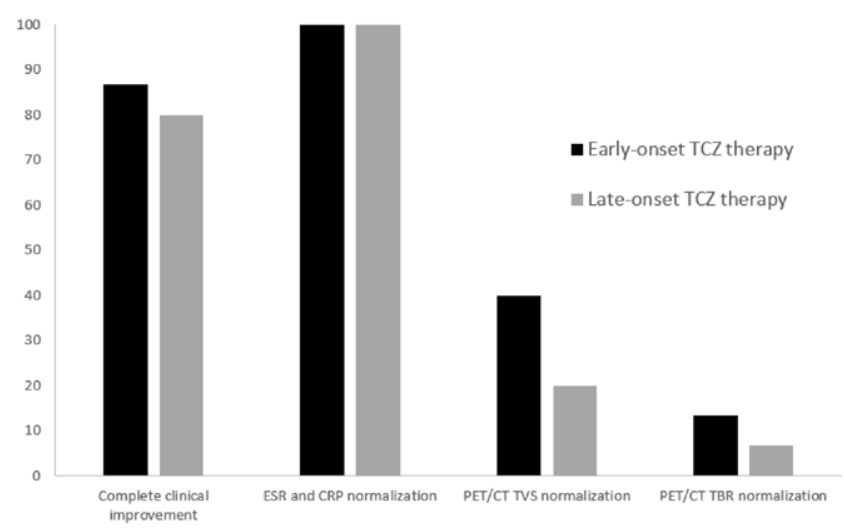

Disclosure of Interests: Diana Prieto-Peña Grant/research support from: UCB Pharma, Roche, Sanofi, Pfizer, AbbVie and Lilly, Isabel Martínez-Rodríguez: None declared, Belén Atienza-Mateo: None declared, Oriana Cuenca-Vera: None declared, Francisco Javier Gomez de la Fuente: None declared, Aida Sanchez-Salmón: None declared, Miguel A González-Gay Grant/research support from: Abbvie, MSD, Jansen and Roche and had consultation fees/participation in company sponsored speaker's bureau from Abbvie, Pfizer, Roche, Sanofi, Lilly, Celgene and MSD, Ricardo Blanco Grant/research support from: Abbvie MSD and Roche, and had consultation fees/participation in company sponsored speaker's bureau from Abbvie, Lilly, Pfizer, Roche, Bristol-Myers, Janssen, UCB Pharma and MSD

DOI: 10.1136/annrheumdis-2021-eular.1733

\section{AB0366 TOCILIZUMAB FOR TAKAYASU ARTERITIS: MULTICENTER STUDY OF 54 WHITE PATIENTS}

D. Prieto-Peña ${ }^{1}$, P. Bernabéu ${ }^{2}$, P. Vela-Casasempere ${ }^{2}$, J. Narváez ${ }^{3}$, C. Fernández-López ${ }^{4}$, M. Freire González ${ }^{4}$, B. González-Alvarez ${ }^{5}$, R. SolansLaqué $^{6}$, J. L. Callejas-Rubio ${ }^{7}$, N. Ortego ${ }^{7}$, C. Fernández-Díaz ${ }^{8}$, E. Rubio Romero $^{9}$, S. García Morillo ${ }^{9}$, M. Minguez ${ }^{10}$, C. Fernández-Carballido ${ }^{10}$, E. De Miguel ${ }^{11}$, S. Melchor ${ }^{12}$, E. Salgado-Pérez ${ }^{13}$, B. Bravo ${ }^{14}$, S. RomeroYuste $^{15}$, J. Salvatierra ${ }^{7}$, C. Hidalgo ${ }^{16}$, S. Manrique Arija ${ }^{17}$, C. Romero-Gómez ${ }^{17}$ P. Moya ${ }^{18}$, N. Alvarez-Rivas ${ }^{19}$, J. Mendizabal ${ }^{20}$, F. M. Ortiz Sanjuan ${ }^{21}$, I. Pérez de Pedro ${ }^{22}$, J. L. Alonso Valdivieso ${ }^{23}$, P. S. Laura ${ }^{24}$, R. M. Rosa ${ }^{24}$, N. FernándezLlanio $^{25}$, R. Gómez de la Torre ${ }^{26}$, S. Suarez ${ }^{26}$, M. J. Montesa ${ }^{5}$, M. Delgado Sanchez $^{5}$, J. Loricera ${ }^{1}$, B. Atienza-Mateo ${ }^{1}$, S. Castañeda ${ }^{8}$, M. A. GonzálezGay ${ }^{1}$, R. Blanco'. ${ }^{1}$ Hospital Universitario Marques de Valdecilla, IDIVAL, Rheumatology, Santander, Spain; ${ }^{2} \mathrm{H}$. General de Alicante, -, Alicante, Spain; ${ }^{3} \mathrm{H}$. Bellvitge,.., Barcelona, Spain; ${ }^{4} \mathrm{CHU}$ A Coruña,.., A Coruña, Spain; ${ }^{5} \mathrm{H}$ Nuestra Sa Candelaria,.., Tenerife, Spain; ${ }^{6}$ H. Vall d'Hebron,.., Barcelona, Spain; ${ }^{7}$ H. San Cecilio,.. Granada, Spain; ${ }^{8} \mathrm{H}$. La Princesa,.. Madrid, Spain; ${ }^{9} \mathrm{H}$. Virgen Rocío,.., Sevilla, Spain; ${ }^{10}$ H. San Juan Alicante,.., Alicante, Spain; ${ }^{11}$ H. La Paz,.., Madrid, Spain; ${ }^{12} \mathrm{H} .12$ Octubre,.., Madrid, Spain; ${ }^{13} \mathrm{CHU}$ Ourense,.., Ourense, Spain; ${ }^{14} \mathrm{H}$. Virgen Nieves,.., Granada, Spain; ${ }^{15} \mathrm{CHU}$ Pontevedra,., Pontevedra, Spain; ${ }^{16} \mathrm{CHU}$ Salamanca,.., Salamanca, Spain; ${ }^{17} \mathrm{H}$. Regional Malaga,.., Malaga, Spain; ${ }^{18} \mathrm{H}$. San Pau,.., Barcelona, Spain; ${ }^{19} \mathrm{H}$. San Agustin,.., Aviles, Spain; ${ }^{20} \mathrm{CH}$ Navarra,.., Pamplona, Spain; ${ }^{21}$ H. La Fe,.. Valencia, Spain; ${ }^{22}$ H. Carlos Haya,. Malaga, Spain; ${ }^{23}$ H. Burgos,., Burgos, Spain; ${ }^{24}$ H. Reina Sofia,., Cordoba, Spain; ${ }^{25}$ H. Arnau Vilanova,.. Valencia, Spain; ${ }^{26}$ H. Central Asturias,.., Oviedo, Spain

Background: Tocilizumab (TCZ) has shown to be effective for large vessel vasculitis including Takayasu arteritis (TAK) (1-3). Most evidence in TAK comes from Asian patients. However, white patients seem to have different clinical and prog nostic features.

Objectives: Our aims were to: a) assess the efficacy and safety of TCZ in white patients with refractory TAK, b) determine if clinical improvement correlates with imaging outcomes, c) compare TCZ in monotherapy $\left(\mathrm{TCZ}_{\mathrm{MONO}}\right)$ vs combined with conventional immunosuppressive drugs ( $\mathrm{TCZ}_{\text {сомво }}$ )

Methods: Multicenter study of white patients with refractory TAK who received TCZ.Outcomes variables were remission, glucocorticoid-sparing effect, improvement in imaging techniques, and adverse events. A comparative study between patients who received $\mathrm{TCZ}_{\mathrm{MONO}}$ and $\mathrm{TCZ}_{\mathrm{COMBO}}$ was performed.

Results: 54 patients ( 46 women/8 men; median age 42.0 [32.5-50.5] years) TCZ was started after 12.0 [3.0-31.5] months since TAK diagnosis. Remission was achieved in 12/54 (22.2\%), 19/49 (38.8\%), 23/44 (52.3\%) and 27/36 (75\%) 
at $1,3,6$ and 12 months, respectively. Prednisone dose was reduced from 30.0 [12.5-50.0] to $5.0[0.0-5.6] \mathrm{mg} /$ day at 12 months (Table 1$) .10(26.3 \%)$ of the 38 patients in whom an imaging follow-up test was performed showed no radiographic improvement after a median of 9.0 [6.0-14.0] months. 4 of them were in clinical remission.23 (42.6\%) patients were on $\mathrm{TCZ}_{\mathrm{MONO}}$ and 31 (57.4\%) on TCZ $:$ MTX $(n=28)$, cyclosporine A $(n=2)$, azathioprine $(n=1)$. Patients on $\mathrm{TCZ}_{\text {Сомво }}$ were younger (38.0 [27.0-46.0] vs 45 [38.0-57.0] years; $p=0.048$ ), with a trend to longer TAK duration (21.0 [6.0-38.0] vs 6.0 [1.0-23.0] months; $\mathrm{p}=0.08)$ and higher C-reactive protein $(2.4$ [0.7-5.6] vs $1.3[0.3-3.3] \mathrm{mg} / \mathrm{dL}$; $p=0.16$ ). Despite these differences, similar outcomes were observed in both groups (log rank $\mathrm{p}=0.862$ ) (Figure 1). Relevant adverse events were reported in $6(11.1 \%)$ patients, but only 3 developed severe events that required TCZ withdrawal.

Table 1.

\begin{tabular}{|c|c|c|c|c|c|}
\hline & $\begin{array}{l}\text { Baseline } \\
\mathrm{n}=54\end{array}$ & $\begin{array}{c}\text { Month } 1 \\
\mathrm{~N}=54\end{array}$ & $\begin{array}{c}\text { Month } 3 \\
\mathrm{~N}=49\end{array}$ & $\begin{array}{c}\text { Month } 6 \\
\mathrm{~N}=44\end{array}$ & $\begin{array}{c}\text { Month } 12 \\
\mathrm{~N}=36\end{array}$ \\
\hline $\begin{array}{l}\text { Clinical remis- } \\
\text { sion, } \mathrm{n}(\%) \\
\text { Laboratory } \\
\text { improvement }\end{array}$ & & $12(22.2)$ & 19 (38.8) & 23 (52.3) & $27(75.0)$ \\
\hline $\begin{array}{l}\mathrm{CRP}(m g / d L), \\
\text { median }[\mathrm{IQR}]\end{array}$ & $\begin{array}{c}1.5 \\
{[0.5-3.5]}\end{array}$ & $\begin{array}{c}0.2 \\
{[0.1-0.7]^{*}}\end{array}$ & $\begin{array}{c}0.2 \\
{[0.5-0.5]^{*}}\end{array}$ & $\begin{array}{l}0.2 \\
{[0.1-0.5]^{*}}\end{array}$ & $\begin{array}{c}0.1 \\
{[0.0-0.4]^{*}}\end{array}$ \\
\hline $\begin{array}{l}\mathrm{ESR}\left(\mathrm{mm} / 1^{\text {st }} \text { hour }\right) \\
\text { median }[\mathrm{IQR}]\end{array}$ & $\begin{array}{l}30.5 \\
{[8.7-52.7]}\end{array}$ & $\begin{array}{c}7.0 \\
{[3.0-14.0]^{*}}\end{array}$ & $\begin{array}{c}4.5 \\
{[2.0-8.0]^{*}}\end{array}$ & $\begin{array}{c}5.0 \\
{[2.0-6.0]^{\star}}\end{array}$ & $\begin{array}{c}4.0 \\
{[2.0-9.5]^{*}}\end{array}$ \\
\hline $\begin{array}{l}\text { Hemoglobin }(g / d L) \\
\text { mean } \pm \mathrm{SD}\end{array}$ & $12.4 \pm 1.5$ & $13.0 \pm 1.2^{*}$ & $13.0 \pm 1.4^{\star}$ & $13.2 \pm 1.5^{\star}$ & $12.9 \pm 1.6^{\star}$ \\
\hline $\begin{array}{l}\text { Prednisone dose, } \\
\text { median [IQR] }\end{array}$ & $\begin{array}{c}30.0 \\
{[12.5-50.0]}\end{array}$ & $\begin{array}{c}20.0[10.0- \\
30.0]^{*}\end{array}$ & $\begin{array}{c}10.0 \\
{[5.0-20.0]^{*}}\end{array}$ & $\begin{array}{l}5.0 \\
{[5.0-10.5]^{\star}}\end{array}$ & $\begin{array}{c}5.0 \\
{[0.0-5.6]^{*}}\end{array}$ \\
\hline
\end{tabular}

CRP: C-Reactive Protein; ESR: Erythrocyte Sedimentation Rate; IQR: interquartile range; $n$ : number. ${ }^{*} p<0.01$ vs baseline (Wilcoxon test).

Conclusion: TCZ is effective and safe in white patients with refractory TAK. A discordance between clinical and imaging activity assessment may exist.

REFERENCES:

[1] Prieto Peña D et al. Clin Exp Rheumatol 2020 Nov 27. PMID: 33253103.

[2] Loricera J, et al. Clin Exp Rheumatol 2016; 34:S44-53. PMID: 27050507.

[3] Calderón-Goercke M, et al. Semin Arthritis Rheum 2019; 49:126-35. PMID: 30655091

FIGURE 1

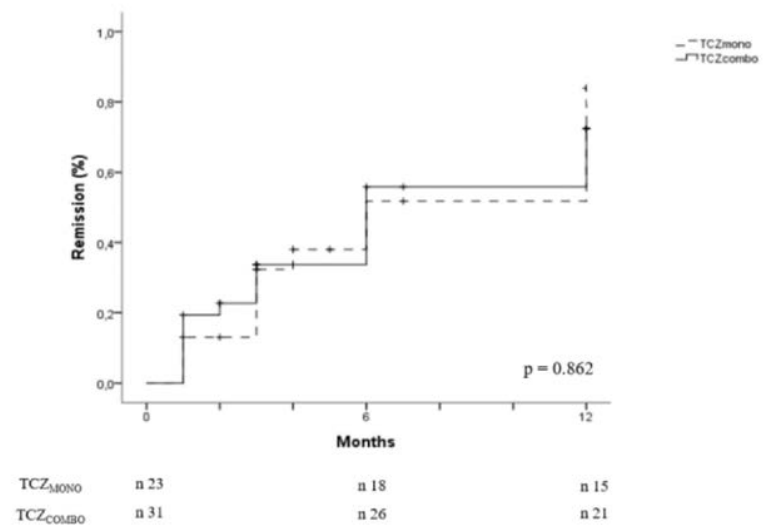

Disclosure of Interests: Diana Prieto-Peña Grant/research support from: DP-P has received research support from UCB Pharma, Roche, Sanofi, Pfizer, AbbVie and Lilly., Pilar Bernabéu: None declared, Paloma Vela-Casasempere: None declared, J. Narváez: None declared, Carlos Fernández-López: None declared, Mercedes Freire González: None declared, Beatriz González-Alvarez: None declared, Roser Solans-Laqué: None declared, Jose Luis Callejas-Rubio: None declared, Norberto Ortego: None declared, Carlos Fernández-Díaz: None declared, Esteban Rubio Romero: None declared, SALVADOR GARCÍA MORILLO: None declared, Mauricio Minguez: None declared, Cristina Fernández-Carballido: None declared, Eugenio de Miguel: None declared, Sheila Melchor: None declared, Eva Salgado-Pérez: None declared, Beatriz Bravo: None declared, Susana Romero-Yuste: None declared, Juan Salvatierra: None declared, Cristina Hidalgo: None declared, Sara Manrique Arija: None declared, C. Romero-Gómez: None declared, Patricia Moya: None declared, Noelia Alvarez-Rivas: None declared, Javier Mendizabal: None declared, Francisco Miguel Ortiz Sanjuan: None declared, I. Pérez de Pedro: None declared, JOSE LUIS ALONSO VALDIVIESO: None declared Pérez Sánchez Laura: None declared, Roldán Molina Rosa: None declared Nagore Fernández-Llanio: None declared, Ricardo Gómez de la Torre: None declared, Silvia Suarez: None declared, María Jesús Montesa: None declared, Monica Delgado Sanchez: None declared, J. Loricera: None declared, Belén Atienza-Mateo: None declared, Santos Castañeda: None declared, Miguel A González-Gay Grant/research support from: MAG-G received grants/research supports from Abbvie, MSD, Jansen and Roche and had consultation fees/participation in company sponsored speaker's bureau from Abbvie, Pfizer, Roche, Sanofi, Lilly, Celgene and MSD, Ricardo Blanco Grant/research support from: RB received grants/research supports from Abbvie, MSD and Roche, and had consultation fees/participation in company sponsored speaker's bureau from Abbvie, Lilly, Pfizer, Roche, Bristol-Myers, Janssen, UCB Pharma and MSD. DOI: 10.1136/annrheumdis-2021-eular.1747

\section{$\mathrm{AB} 0367$ \\ DIAGNOSTIC DELAY IN PATIENTS WITH GIANT CELL ARTERITIS}

M. Van Nieuwland ${ }^{1}$, E. Colin ${ }^{1}$, D. Boumans ${ }^{1}$, M. Vermeer $^{2}$, E. Brouwer ${ }^{3}$ C. Alves ${ }^{1}$. ${ }^{1}$ Hospital Group Twente, Rheumatology and Clinical Immunology, Almelo, Netherlands; ${ }^{2}$ Hospital Group Twente, ZGT Academy, Almelo, Netherlands; ${ }^{3}$ University Medical Center Groningen, Rheumatology and Clinical Immunology, Groningen, Netherlands

Background: Giant cell arteritis (GCA) is the most common systemic vasculitis in the elderly which can lead to severe complications when treatment is delayed. Therefore, timely diagnosis and start of treatment is essential. Sev eral forms of delay (consultation, referral and diagnostic delay) can contribute to the total delay towards GCA diagnosis. In the Dutch healthcare system, treatment is not started by a general practitioner (GP). When GCA is suspected, GPs refer to specialists. In our hospital, a fast track clinic (FTC) is used to expedite diagnosis. However, information on factors contributing to delay is scarce.

Objectives: The aim of this study was to describe the different components of delay towards diagnosis in GCA suspected patients in a general hospital, Ziekenhuisgroep Twente (the Netherlands).

Methods: For this descriptive study, a retrospective cohort consisting of patients with suspected GCA between January $1^{\text {st }} 2017$ and October $1^{\text {st }} 2019$ was used to analyse components of delay in diagnosis, as suggested by Prior et al. Consultation delay was defined as the time between start of symptoms and a patient's first consultation with a GP. Referral delay was defined as the time between a patient's first consultation with a GP and first visit to the FTC. Diagnostic delay was defined as the time between the first visit to the FTC and treatment initiation. Total delay was defined as the time from symptom onset until start of treatment. Delays were described using the median and interquartile ranges (IQR).

Results: In our cohort, 206 patients were included for analysis of whom 62 had GCA. Controls $(n=144)$ were suspected of but did not have GCA. Comparing GCA patients with controls, $66.1 \%$ and $50.7 \%$ were female and the mean (SD) age was $74.2(9.4)$ and 70.2 (11.0) years, respectively. In our cohort, the majority of patients $(n=42,67.7 \%)$ had cranial GCA (C-GCA). Furthermore, $8(12.9 \%)$ had large vessel GCA (LV-GCA) and $12(19.4 \%)$ had a combination of C-GCA and LV-GCA. For GCA patients, median consultation delay was 2.1 (IQR 0.8-5.8) weeks, referral delay 1.4 (IQR 0.4-4.6) weeks and diagnostic delay 0 (IQR 0-0.1) weeks (Figure 1). For delay regarding consultation and referral, results of controls were comparable to GCA patients. The median total delay was 4.4 weeks (IQR 1.57-10.14) for GCA patients.

Conclusion: With a median total delay of 4.4 weeks, delay in our cohort is almost half the delay described in a review by Prior et al. This difference might be due to FTC implementation and subsequent awareness in our hospital and by local GPs. Patients generally received treatment within one day after FTC visit. Nevertheless, contribution of consultation and referral delay is not resolved by introduction of the FTC, as shown in our data. Timely diagnosis is essential as severe complications can develop instantly, which emphasizes the need to tackle consultation and referral delay.

\section{REFERENCES:}

[1] Prior JA, Ranjbar H, Belcher J, et al. Diagnostic delay for giant cell arteritis - a systematic review and meta-analysis. BMC Med. 2017;15(1):120. 